\title{
Divergent effects of irradiation on brain cortical morphology in patients with nasopharyngeal carcinoma: one-year follow-up study using structural magnetic resonance imaging
}

\author{
Xiaofei Lv ${ }^{1 \#}$, Zheng Guo ${ }^{2 \#}$, Linquan Tang ${ }^{3}$, Zhipeng $\mathrm{Li}^{1}$, Xiaoshan Lin $^{4}$, Jing Li $^{1}$, Lujun Han ${ }^{1}$, \\ Yingwei Qiu ${ }^{4}$, Haiqiang Mai ${ }^{3}$
}

${ }^{1}$ Department of Medical Imaging, Sun Yat-sen University Cancer Center, State Key Laboratory of Oncology in South China, Collaborative Innovation Center for Cancer Medicine, Guangdong Key Laboratory of Nasopharyngeal Carcinoma Diagnosis and Therapy, Guangzhou, China; ${ }^{2}$ Department of Oncology, The First Affiliated Hospital of Ganzhou Medical University, Ganzhou, China; ${ }^{3}$ Department of Nasopharyngeal Carcinoma, Sun Yat-sen University Cancer Center, State Key Laboratory of Oncology in South China, Collaborative Innovation Center for Cancer Medicine, Guangdong Key Laboratory of Nasopharyngeal Carcinoma Diagnosis and Therapy, Guangzhou, China; ${ }^{4}$ Department of Radiology, The Third Affiliated Hospital of Guangzhou Medical University, Guangzhou Medical University, Guangzhou, China

\#These authors contributed equally to this work.

Correspondence to: Haiqiang Mai, PhD. Department of Nasopharyngeal Carcinoma, Sun Yat-sen University Cancer Center, State Key Laboratory of Oncology in South China, Collaborative Innovation Center for Cancer Medicine, Guangdong Key Laboratory of Nasopharyngeal Carcinoma Diagnosis and Therapy, 651 Dongfeng Road East, Guangzhou 510060, China. Email: maihq@sysucc.org.cn; Yingwei Qiu, PhD. Department of Radiology, The Third Affiliated Hospital of Guangzhou Medical University, Guangzhou Medical University, No. 63 Duobao Road, Liwan District, Guangzhou 510150, China. Email: qiuyw1201@gmail.com.

Background: Increasing evidence indicates that radiotherapy (RT)-induced brain cortical deficits may play a critical role in developing radiation encephalopathy in patients with nasopharyngeal carcinoma (NPC). However, the evolutional processes of RT-induced cortical injury have not been sufficiently investigated. This study investigates RT-induced effects on cortical morphology using longitudinal structural magnetic resonance imaging (MRI) in NPC patients.

Methods: Using MRI-based morphometry with surface-based measures, we evaluated the longitudinal alterations of cortical volume (CV), cortical thickness (CT), and cortical surface area (CSA) in 104 NPC patients at pre-RT ( $\mathrm{n}=104)$, within 3 months post-RT ( $\mathrm{n}=92), 6$ months post-RT ( $\mathrm{n}=71)$, and 9-12 months post-RT (n=52). Twenty healthy controls were also evaluated in parallel. Linear mixed models were used to investigate the trajectories of RT-related changes in cortical brain morphology and its association with irradiation dose, with healthy controls data being used to construct a normal age-related benchmark. The level of statistical significance was set at $\mathrm{P}<0.05$, corrected for multiple comparisons.

Results: The results showed that RT-related longitudinal alterations in cortical morphology underwent two diverse patterns during the first year of follow up in NPC patients. The temporal cortices (including the bilateral superior temporal gyrus, middle temporal gyrus, temporal pole, parahippocampal and fusiform gyrus, and the right inferior temporal and right transverse temporal gyrus), the basal occipital cortices (the right lingual gyrus and lateral occipital gyrus), and the basal frontal cortices (the right lateral orbitofrontal gyrus) showed time-dependent attenuation in cortical morphology indices. Furthermore, these effects on multiple cortices were dose-dependent, suggesting they were RT-associated. In contrast, in the left rostral middle frontal gyrus, there was a time-dependent increase in CT.

Conclusions: Our preliminary findings revealed divergent effects of irradiation on cortical brain morphology. These results contribute to a more comprehensive understanding of the underlying neural mechanisms of irradiation-related neurotoxic effects on cortical brain morphology and will help guide the investigation of critically neuroprotective strategies. 


\begin{abstract}
Keywords: Cortical thickness (CT); cortical surface area (CSA); cortical volume (CV); nasopharyngeal carcinoma
\end{abstract} (NPC); radiotherapy (RT)

Submitted May 14, 2020. Accepted for publication Jan 05, 2021.

doi: 10.21037/qims-20-662

View this article at: http://dx.doi.org/10.21037/qims-20-662

\section{Introduction}

Nasopharyngeal carcinoma (NPC) is one of the most common malignancies of the head and neck in Southeast Asia and Southern China (1), and radiotherapy (RT) has been the mainstay of treatment for non-disseminated NPC (2). However, radiation-induced toxicities for longterm survivors have become an important concern, and radiation-induced brain injury is a major complication. Due to the complexity of radiation effects, brain injury related to irradiation remains poorly understood, and effective prevention or long-term treatment is yet to be established (3). Identifying accurate and sensitive biomarkers of early RT-induced injury in normal-appearing brain tissue in patients with NPC is necessary to facilitate early intervention and guide modification of RT planning strategies that may prevent or minimize brain damage.

Traditionally, radiation damage has been thought to mainly affect the brain's white matter (4-6). However, increasing evidence from magnetic resonance imaging (MRI)-based cortical morphology studies suggest RTinduces changes in the cortex as well (6-11). Dosedependent cortical volume (CV) decrease, especially in the bilateral temporal lobe cortices, has been reported in previous studies $(6,9,11)$ using volumetric measurement, such as the voxel-based morphometry (VBM) method $(6,9)$. However, these results' interpretation can be difficult, given these studies did not measure an actual physical characteristic $(12,13)$. Also, these results may be confounded by the differential effects of their constituents: the cortical thickness (CT) and the cortical surface area (CSA) (14). Currently, surface-based morphometry (SBM) offers the possibility to analyze CT and CSA separately quantitatively $(15,16)$, although, to the best of our knowledge, only two cross-sectional studies $(7,8)$ have documented CT changes in NPC patients following RT. Using a vertex-wise SBM approach, our previous study (8) observed significantly decreased CT in the precentral gyrus of NPC patients at the early-delayed stage post-RT, while we found increased $\mathrm{CT}$ in widespread brain regions at the late-delayed stage.
However, in another study, Zhang et al. (7) found a significant reduction in CT and an increase in the CSA in post-RT patients' temporal lobes compared with the CT and CSA of pre-RT patients with NPC. These inconsistent and conflicting findings must be interpreted with caution as a cross-sectional study design can be influenced by betweensubject variance and possible cohort effects, as well as an inability to detect intra-individual alterations. Therefore, longitudinal studies are needed to dissociate these intra- and inter-individual differences.

In the present study, we evaluated one-year longitudinal alterations in the brain CV, CT, and CSA of NPC patients treated with RT to obtain a more comprehensive understanding of the pathophysiology of radiationinduced brain cortical injuries over time and to identify brain cortical subregions with particular vulnerability to radiation damage as part of RT planning. Based on prior studies $(7,8)$, we hypothesized that RT-induced dynamic alterations in cortical morphology (CV/CT/CSA) could be accurately detected in vivo and used as a noninvasive biomarker for the evaluation of RT-induced neurotoxicity in the normal-appearing brain. Also, we expected that longitudinal RT-induced brain CT and CSA changes are time- and dose-dependent; moreover, RT-induced cortical injury may involve specific brain regions due to divergent irradiation sensitivity and inconsistency in irradiation dose distribution (17).

\section{Methods}

\section{Participants}

The Institutional Review Board of the Sun Yat-sen University Cancer Center (Guangzhou, China) reviewed and approved this prospective study, and written informed consent was obtained from each participant. Between December 2014 and January 2019, a total of 104 patients with pathologically-confirmed, treatment-naïve NPC (21-65 years, 64 males and 40 females) and 20 comparable healthy controls (26-55 years, 13 males and 7 females) were 
Table 1 Demographic and clinical characteristics of patients with nasopharyngeal carcinoma and healthy controls at baseline

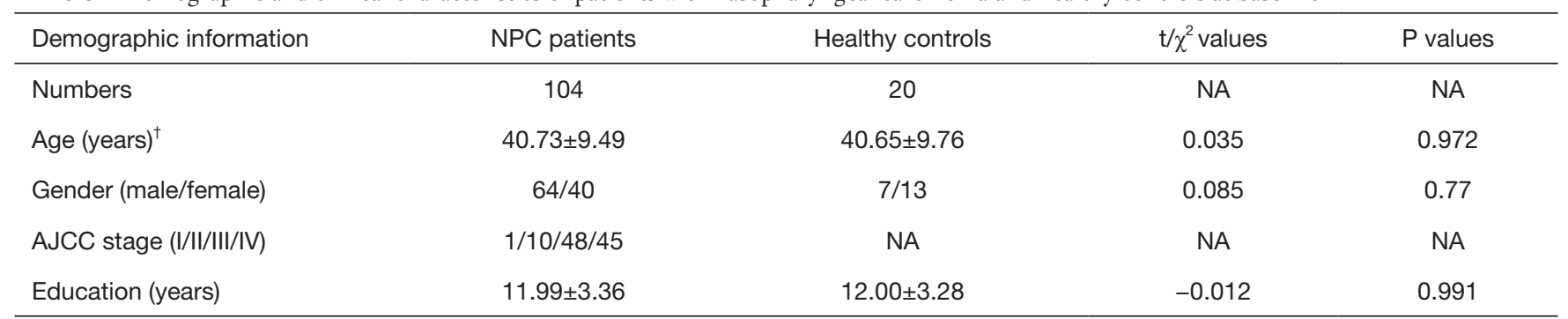

${ }^{\dagger}$ Data are mean \pm standard deviation. AJCC, American Joint Committee on Cancer; RT, radiotherapy; NA, not available; NPC, nasopharyngeal carcinoma.

recruited. Each patient underwent a detailed pretreatment evaluation to determine their clinical stage of NPC, which was defined according to the American Joint Committee on Cancer (AJCC) system (7th edition) (18). The exclusion criteria for all participants were the following: age below 18 years or above 65 years, brain tumors, neurological or psychiatric diseases, prior substantial head trauma, diabetes, viral hepatitis, positive human immunodeficiency virus status, other major illnesses, left-handedness, alcoholism, and contraindications to MRI examination. There were additional exclusion criteria for NPC patients, namely a Karnofsky performance status (KPS) score lower than 80 , intracranial invasion, and distant metastases. The demographic and clinical information of all participants is listed in Table 1.

\section{Treatment}

All NPC patients were treated at our institution with intensity-modulated radiation therapy (IMRT) $(n=96)$ or tomography radiation therapy $(\mathrm{n}=8)$ as previously described (19-21). Dose evaluation was performed based on the dosevolume histogram for the target brain regions and organs at risk outlined for dose constraint evaluation (19). The main evaluation parameters were the maximum (Dmax), mean (Dmean), and minimum dose (Dmin) received by the bilateral frontal, temporal, and occipital lobes (Table 2). A detailed description of the RT techniques and the treatment protocol is presented in Appendix 1.

\section{Follow-up procedure}

To assess the dynamic alteration of cortical brain morphology in NPC patients after RT, all patients were longitudinally followed up between two and four times at the following time points: before treatment initiation (baseline, $\mathrm{n}=104)$, within 3 months ( $\mathrm{n}=92), 6$ months $(\mathrm{n}=71)$, and 9-12 months $(\mathrm{n}=52)$ following RT completion. Brain MRI scans were acquired for these patients at each followup time point. The 20 healthy controls were followed up in parallel (baseline, 6 months, 9 months, and 15 months) and MRI examinations were performed at each time point. In total, we collected 319 and 80 MRI data for NPC patients and healthy controls, respectively. The schedule of the follow-up measurements for all participants is shown in Figure 1.

\section{MRI acquisition}

All participants underwent MR scan using a 3.0 Tesla MRI scanner with a 16-channel head and neck coil (Discovery MR750; General Electric, Milwaukee, WI, USA) at the Sun Yat-sen University Cancer Center. To detect intracranial lesions, conventional MR images, including axial T1- and T2-weighted images and T2-FLAIR images were acquired for each participant. High-resolution T1-weighted structural MR images were then collected using a three-dimensional brain volume imaging (3D-BRAVO) sequence. The imaging parameters were as follows: $\mathrm{TR} / \mathrm{TE}=8.16 / 3.18 \mathrm{~ms}$, inversion time $=800 \mathrm{~ms}$, flip angle $=8^{\circ}$, Field of View $=256 \times 256 \mathrm{~mm}^{2}$, acquisition matrix $=256 \times 256 \mathrm{~mm}^{2}$, slice thickness $=1 \mathrm{~mm}$, no inter-slice gap, voxel size $=1 \times 1 \times 1 \mathrm{~mm}^{3}$, and 176 sagittal slices.

\section{Image processing}

The raw data acquired from 3D-BRAVO sequences was used to analyze cortical morphology. Preprocessing of the structural images was performed using the open-source FreeSurfer pipeline (22-25) (version 6.0, http://surfer.nmr. mgh.harvard.edu) as previously conducted in our laboratory $(11,26)$. First, we obtained within-subject templates from 
Table 2 Dose-volume statistics of the bilateral temporal lobe for 104 patients with nasopharyngeal carcinoma treated with radiation therapy (Gy)

\begin{tabular}{|c|c|c|c|}
\hline Brain regions & Maximum dose (Dmax) & Minimum dose (Dmin) & Mean dose (Dmean) \\
\hline The right temporal lobe & $69.15 \pm 7.68$ & $2.02 \pm 3.26$ & $17.53 \pm 6.62$ \\
\hline The left occipital lobe & $20.97 \pm 7.62$ & $1.33 \pm 0.56$ & $6.47 \pm 2.99$ \\
\hline The right occipital lobe & $21.46 \pm 7.80$ & $1.34 \pm 0.45$ & $6.63 \pm 3.00$ \\
\hline The Right frontal lobe & $15.83 \pm 17.70$ & $1.63 \pm 0.54$ & $3.85 \pm 3.20$ \\
\hline
\end{tabular}

All data are presented as mean \pm standard deviation.

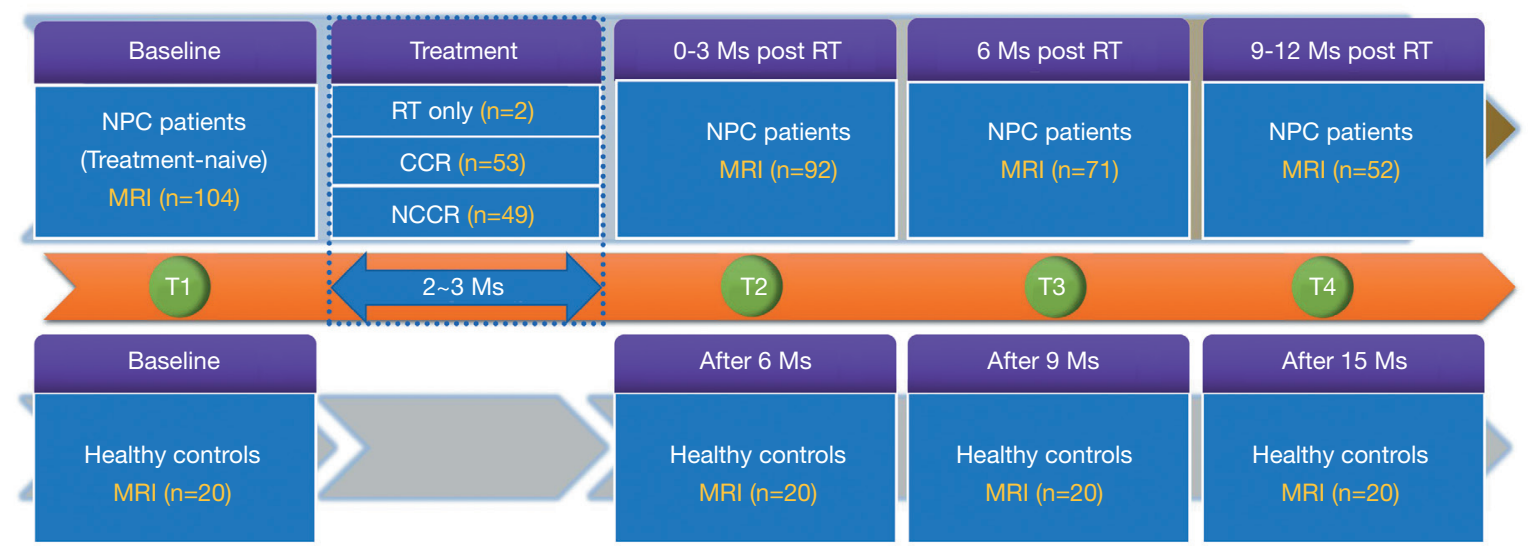

Figure 1 Enrollment and follow-up procedures for patients with NPC and healthy controls. CCR, concurrent chemoradiotherapy; RT, radiotherapy; Ms, months; NCCR, neoadjuvant/adjuvant chemotherapy combined with concurrent chemoradiotherapy; NPC, nasopharyngeal carcinoma; $\mathrm{T}$, time-point.

images of individual participants at pretreatment and all post-treatment time points. We then processed longitudinal data using a specialized longitudinal processing stream, which can reduce variability in within-subject morphological measures, resulting in higher statistical power and allowing the detection of small changes (27). The CV, CT, and CA values for each of the 68 parcels defined by the Desikan parcellation were extracted for each subject and at each time point (28). Estimated total intracranial volume (29,30) (TIV) was used as a covariate in all correlational analyses involving brain variables to compensate for inter-individual differences in head size.

\section{Statistical analyses}

Open-source R software (version 3.1.3) was used for all analyses.

\section{Demographic analysis}

We tested any demographic differences between the NPC patients and healthy controls at baseline using the chisquared test for categorical variables (gender) and twosample independent $t$-tests for continuous variables (age and education). A $\mathrm{P}$ value of less than 0.05 was considered significant.

\section{Longitudinal brain morphometric trajectory in NPC patients post-RT}

Linear mixed models were used to investigate RT-related brain morphometric changes' trajectories and their association with irradiation dose, with data of healthy controls used to construct a normal age-related benchmark. 
The volume change was modeled linearly, as morphological trajectories in studies of gray matter in NPC patients post-RT are linear (11). As described previously $(11,31)$, random intercepts, as well as random slopes for the effect of time (months since RT or baseline), were modeled for each subject to account for inter-individual variability at baseline and differences in the rate of change following RT (32-34). We constructed two models to elucidate the RT-related brain morphometric changes longitudinally. First, we sought to determine whether there were different trajectories of brain morphometric changes between RTrelated and ageing-related (interaction effect between RT status and time) changes. We accounted for the effects of age at baseline, gender, years of education, and total intracranial volume by modeling these variables as fixed effects. After fitting the model, fixed effects included the RT status, time (months post-RT for NPC or months from the baseline for controls), and their interaction between time post-RT and RT status, education, TIV, and gender. Random effects included the individual intercept and the slope of time as follows:

$Y_{i j}=\gamma_{00}+\gamma_{01}\left(\right.$ gender $\left._{j}\right)+\gamma_{02}\left(\right.$ education $\left._{j}\right)+\gamma_{03}\left(\right.$ age $\left._{j}\right)+\gamma_{04}$ $\left(\right.$ TIV $\left._{\mathrm{j}}\right)+\gamma_{10}\left(\right.$ time $\left._{\mathrm{ij}}\right)+\gamma_{11}\left(\mathrm{RT} \mathrm{status}_{\mathrm{j}} \times\right.$ time $\left._{\mathrm{ij}}\right)+\mu_{0 \mathrm{j}}+\mu_{1 \mathrm{j}}($ time ij) $+r_{i j} \cdot$

In this equation, $Y_{\mathrm{ij}}$ is the brain morphological measurements for the $i$ th participant on the $j$ th followup visit after RT; the longitudinal variable represents the time interval after completion of RT (for NPC patients) or from baseline (for controls); the pretreatment session with quality structural MRI data of each participant is defined as baseline; time always started from zero; the longitudinal time-dependent effect is expressed as a simple regression between time and Y, plus a residual $r$; gender (male or female) and RT status (with RT or without RT) are binary dummy variables; age and education are the grand-meancentered versions of the respective variables. Next, for brain morphological longitudinal changes that showed significantly different trajectories between NPC post-RT and healthy controls, we examined the longitudinal RTrelated changes in these brain measurements with the following linear mixed model:

$Y_{i j}=\gamma_{00}+\gamma_{01}\left(\right.$ gender $\left._{j}\right)+\gamma_{02}\left(\right.$ education $\left._{j}\right)+\gamma_{03}\left(\right.$ age $\left._{j}\right)+\gamma_{04}$ $\left(T_{I V}\right)+\gamma_{10}\left(\right.$ time $\left._{i j}\right)+\mu_{0 j}+\mu_{1 j}\left(\right.$ time $\left._{i j}\right)+r_{i j}$.

The Holm Bonferroni method controlling for familywise errors at alpha level $(0.05)$ was applied to address the multiple testing for the various hypotheses.
Association between longitudinal brain changes and irradiation dose

For brain measurements that showed significant RTrelated longitudinal changes, we examined how these were associated with the corresponding brain regions' irradiation dose. To do this, we calculated the rate of change/per month in brain morphology using the following formula: (brain morphology follow-up - brain morphology baseline)/(brain morphology baseline $\times$ months post-RT). Next, regression analyses were used to examine the association between brain morphological changes and the irradiation dose. Age, sex, and education were added as covariates.

\section{Results}

\section{Demographic and clinical information at baseline}

The demographic and clinical information of NPC patients and healthy controls at baseline are present in Table 1. No significant differences in age, gender, and education level were found between the two groups.

\section{Longitudinal cortical morphology alterations in NPC patients during the first year post-RT}

In the first model, with the data of healthy controls serving as a normal age-related benchmark, NPC patients showed a steeper decrease in a $\mathrm{CV}$ in some sub-regions of the temporal, occipital, and frontal lobes, including the bilateral superior temporal gyrus, middle temporal gyrus, temporal pole, parahippocampal gyrus, and fusiform gyrus, the right inferior temporal gyrus, the right transverse temporal gyrus, the right lateral occipital gyrus, and the right lateral orbitofrontal gyrus. The NPC patients also showed a steeper decrease in CT and CSA in the temporal subregions, including the left superior temporal gyrus and the CT's bilateral temporal pole. The left transverse temporal gyrus, the right inferior temporal gyrus, lingual gyrus, and the parahippocampal gyrus in CSA. In contrast, the left rostral middle frontal gyrus showed a steeper increase in CT in NPC patients (Figure 2). Furthermore, in the second model, patients with NPC had significant time-dependent morphology changes in all the significant regions seen in the first model. Specifically, two time-dependent patterns were observed. In pattern 1 , the temporal cortices (including the superior temporal gyrus, middle temporal gyrus, inferior temporal gyrus, temporal pole, transverse temporal 

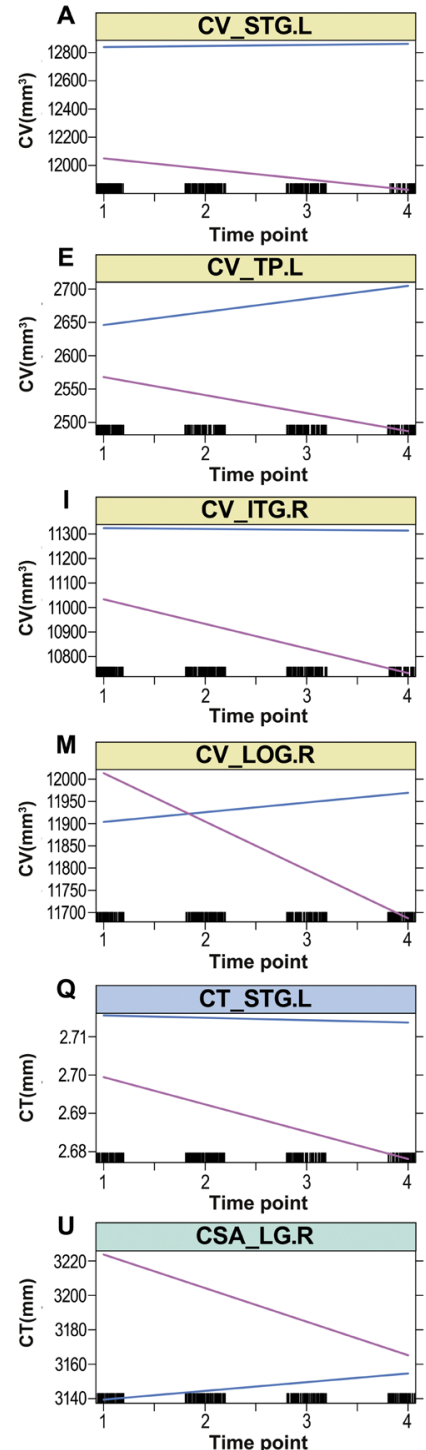
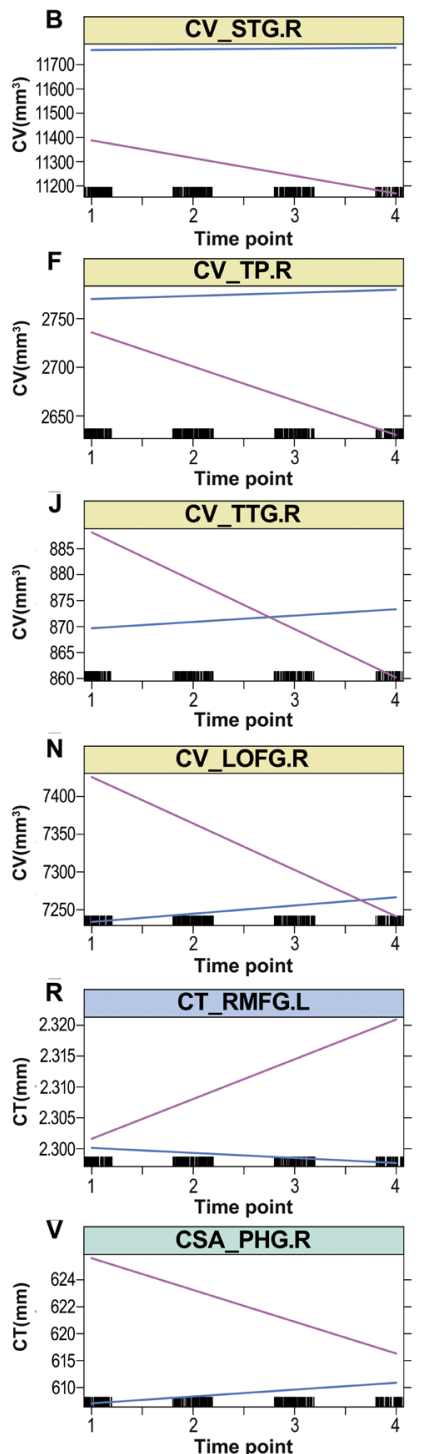
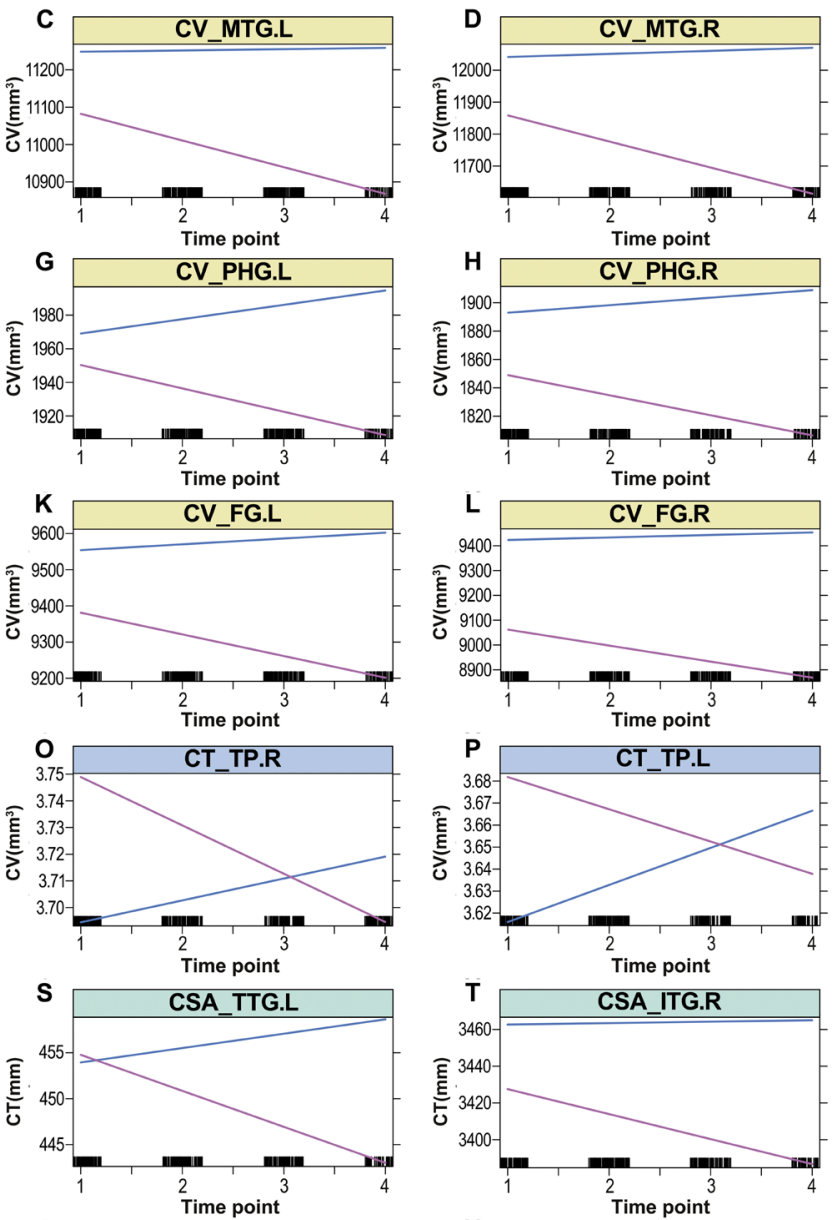

HC group

NPC group

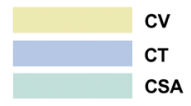

Figure 2 Linear mixed effects model revealed a significant group $\times$ time interaction for cortical morphology in multiple brain regions during 12-months follow-up. Using healthy control data as normal age-related benchmark, NPC patients showed a steeper decrease in $\mathrm{CV}$ in some sub-regions of the temporal, occipital, and frontal lobe (A-N); and steeper decrease in CT and CSA in the temporal subregions, while steeper increase in CT was observed in the left rostral middle frontal gyrus in NPC patients (O-V). CV, cortical volume; CT, cortical thickness; CSA, cortical surface area; STG, superior temporal gyrus; MTG, middle temporal gyrus; TP, temporal pole; PHG, parahippocampal gyrus; ITG, inferior temporal gyrus; FG, fusiform gyrus; LOG, lateral occipital gyrus; LOFG, lateral orbitofrontal gyrus; RMFG, rostral middle frontal gyrus; TTG, transverse temporal gyrus; LG, lingual gyrus; L, left; R, right. 
Table 3 Cortical morphology with significant group $\times$ time interaction in the first model showed time (post-RT) dependent alterations in NPC patients during the first year post RT, corrected for total intracranial volume (TIV), education, and gender in patients with nasopharyngeal carcinoma

\begin{tabular}{|c|c|c|}
\hline Brain region & Coefficient & $\mathrm{P}$ \\
\hline CV_STG.L & -73.97 & $1.64 \mathrm{E}-12^{*}$ \\
\hline CV_STG.R & -73.01 & $1.40 \mathrm{E}-13^{*}$ \\
\hline CV_MTG.L & -71.53 & $4.39 \mathrm{E}-10^{*}$ \\
\hline CV_MTG.R & -81.74 & $9.68 \mathrm{E}-11^{*}$ \\
\hline CV_TP.L & -27.19 & $2.46 \mathrm{E}-14^{*}$ \\
\hline CV_TP.R & -35.3 & $<1.00 \mathrm{E}-15^{\star}$ \\
\hline CV_PHG.L & -13.84 & $7.83 \mathrm{E}-09^{*}$ \\
\hline CV_PHG.R & -14.18 & $3.78 \mathrm{E}-08^{*}$ \\
\hline CV_ITG.R & -99.87 & $8.35 \mathrm{E}-14^{*}$ \\
\hline CV_TTG.R & -9.32 & $1.98 \mathrm{E}-12^{*}$ \\
\hline CV_FG.L & -59.77 & $2.61 \mathrm{E}-09^{*}$ \\
\hline CV_FG.R & -64.41 & $3.04 \mathrm{E}-11^{*}$ \\
\hline CV_LOG.R & -110.45 & $2.44 \mathrm{E}-07^{*}$ \\
\hline CV_LOFG.R & -61.09 & $1.69 \mathrm{E}-07^{*}$ \\
\hline CT_TP.L & -0.02 & $6.20 \mathrm{E}-05^{\star}$ \\
\hline CT_TP.R & -0.02 & $1.41 \mathrm{E}-05^{\star}$ \\
\hline CT_STG.L & -0.01 & $1.59 \mathrm{E}-13^{*}$ \\
\hline CT_RMFG.L & 0.01 & $2.24 \mathrm{E}-07^{*}$ \\
\hline CSA_TTG.L & -3.93 & 3.36E-12* \\
\hline CSA_ITG.R & -13.63 & $9.30 \mathrm{E}-13^{*}$ \\
\hline CSA_LG.R & -19.5 & $2.13 \mathrm{E}-11^{*}$ \\
\hline CSA_PHG.R & -2.35 & $5.67 \mathrm{E}-07^{*}$ \\
\hline
\end{tabular}

*Statistically significant effects $(P<0.05)$. CV, cortical volume; CT, cortical thickness; CSA, cortical surface area; STG, superior temporal gyrus; MTG, middle temporal gyrus; TP, temporal pole; PHG, parahippocampal gyrus; ITG, inferior temporal gyrus; FG, fusiform gyrus; LOG, lateral occipital gyrus; LOFG, lateral orbitofrontal gyrus; RMFG, rostral middle frontal gyrus; TTG, transverse temporal gyrus; LG, lingual gyrus; L, left; R, right.

gyrus, parahippocampal gyrus, and fusiform gyrus), the occipital cortex (lingual gyrus and lateral occipital gyrus), and the frontal cortex (lateral orbitofrontal gyrus) showed time-dependent attenuation in cortical morphology indices (CV, CT and/or CSA) (Table 3). In pattern 2 the left rostral middle frontal gyrus showed a time-dependent CT increase (Table 3). Given that most NPC patients were treated with IMRT, we also repeated the above analysis in NPC patients with IMRT, and the results were similar to the primary results, as shown in the Table $\mathrm{S} 1$.

\section{Association between irradiation dose and changes in $\mathrm{CV}$, $C T$, and CSA in the NPC group}

Significant negative correlations were found between the mean dose to the ipsilateral temporal lobe and $\mathrm{CV}$ changes in the left fusiform gyrus, the left parahippocampal gyrus, and the right transverse temporal gyrus; CT changes of the left superior temporal gyrus; and CSA changes of the right inferior temporal gyrus (Figure 3). Significant negative correlations were also observed between $\mathrm{CV}$ changes in the right lateral occipital gyrus and the mean dose to the left occipital lobe and between $\mathrm{CV}$ changes in the right lateral orbitofrontal gyrus and the mean dose to the left frontal lobe (Figure 3).

\section{Discussion}

This is the first longitudinal study to track changes in cortical brain morphology (CV, CT, and CSA) using an SBM approach in a relatively large sample of patients with NPC at multiple time points over 12 months after RT, and with healthy control, data serving as a normal agerelated benchmark. Our results revealed that RT-related longitudinal alterations in cortical morphology underwent two diverse patterns during the first year follow-up in NPC patients. In pattern 1 , the temporal cortices (including the superior temporal gyrus, middle temporal gyrus, inferior temporal gyrus, temporal pole, transverse temporal gyrus, parahippocampal gyrus, and fusiform gyrus), the basal occipital (lingual gyrus and lateral occipital gyrus), and the basal frontal cortices (lateral orbitofrontal gyrus) showed time-dependent attenuation in cortical morphology indices. In pattern 2, the left rostral middle frontal gyrus showed a time-dependent increase in CT. Furthermore, the reduction in the cortical morphology of the temporal (the left fusiform gyrus, parahippocampal gyrus, and superior temporal gyrus, the right transverse temporal gyrus and inferior temporal gyrus), basal occipital, and basal frontal cortices (the right lateral occipital gyrus and lateral orbitofrontal gyrus) was dose-dependent. These findings will lead to a better understanding of the neural mechanisms underlying 

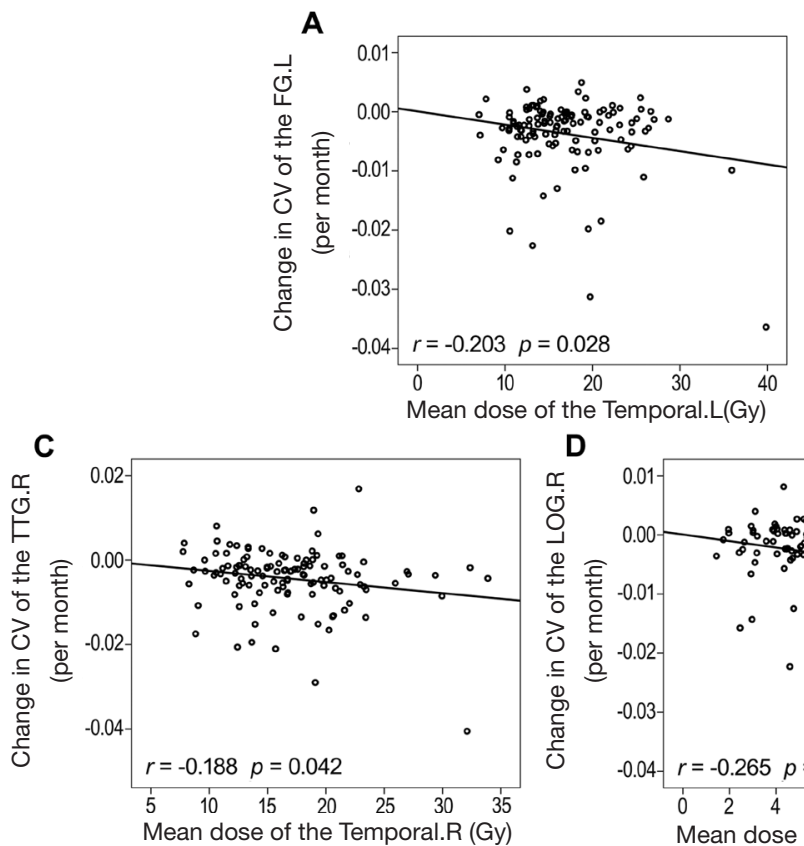

$$
\mathbf{F}
$$

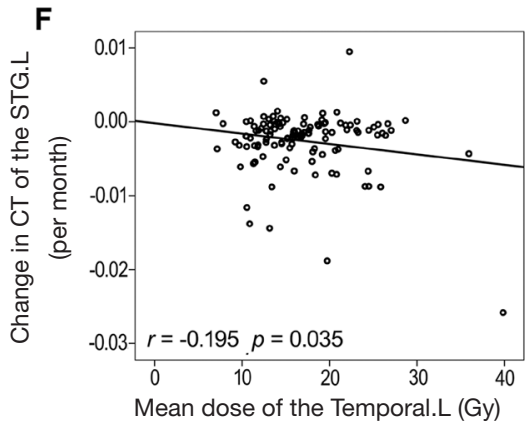

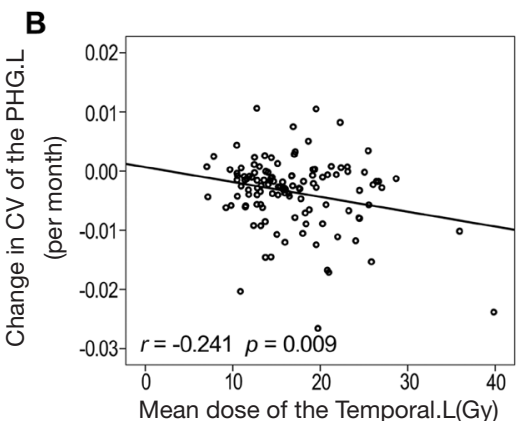
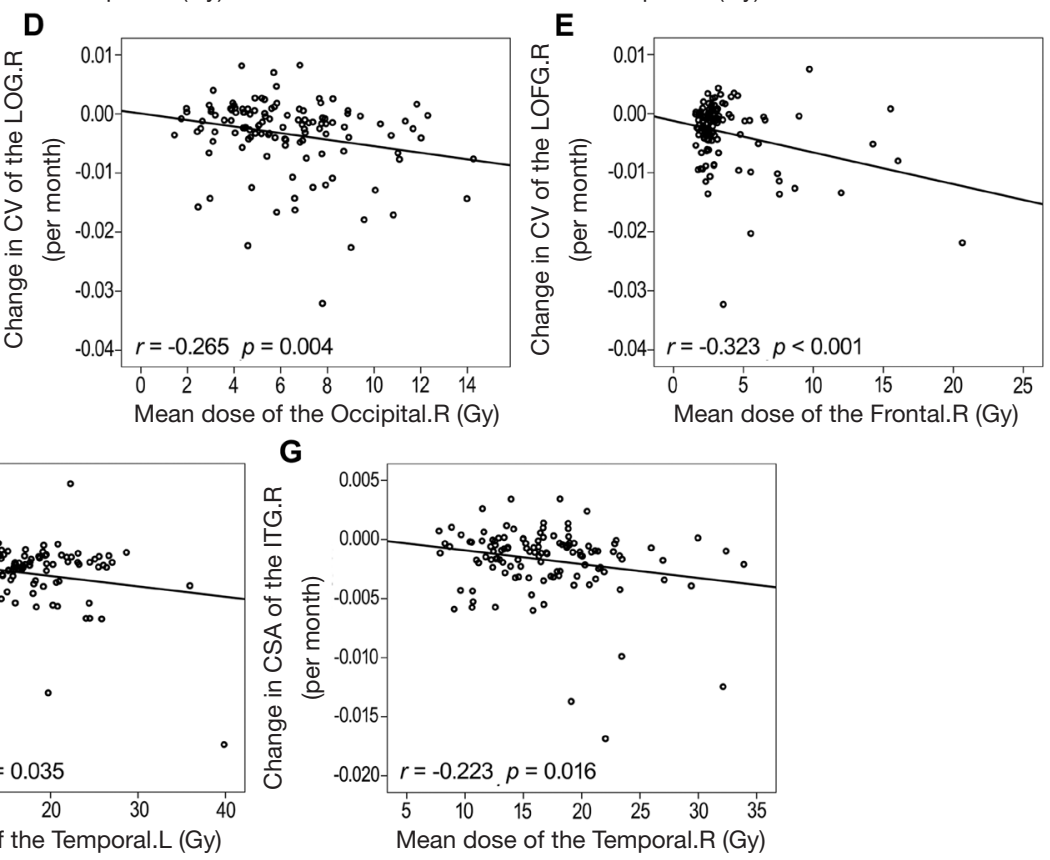

Figure 3 Correlation between changes in cortical morphology (CV/CT/CSA) and radiation dosage. Dose-dependent volume reductions of the left fusiform gyrus (A), the left parahippocampal gyrus (B), the right transverse temporal gyrus (C), the right lateral occipital gyrus (D), the right lateral orbitofrontal gyrus (E), dose-dependent reductions of CT in the left superior temporal gyrus (F), and the dose-dependent reductions of CSA in the right inferior temporal gyrus $(\mathrm{G})$ were observed. Each point represents the subject-level longitudinal change in cortical morphology (CV/CT/CSA) (y-axis; higher values indicate greater loss) and the corresponding mean dose to the brain regions (x-axis). CV, cortical volume; CT, cortical thickness; CSA, cortical surface area; FG, fusiform gyrus; PHG, parahippocampal gyrus; TTG, transverse temporal gyrus; LOG, lateral occipital gyrus; LOFG, lateral orbitofrontal gyrus; L, left; R, right.

radiation-induced brain injuries.

\section{RT-induced cortical morphology changes in the temporal, occipital, and frontal lobes}

We found time-dependent reductions in the $\mathrm{CV}$ of multiple temporal cortices in NPC patients during the first year after RT. The present results are in line with previous MRI-based studies, both in the cross-sectional $(6,9)$ and the longitudinal design (11), which also showed radiation- induced temporal cortices atrophy in NPC patients. This finding is unsurprising, given that these regions are anatomically all close to or are overlapped by the clinical target volume, which receives unavoidable radiation exposure, which can lead to radiation damage. Furthermore, we provided additional dynamic information (time after RT) on radiation-induced temporal cortices volumetric changes over a relatively long period (12 months) post-RT after controlling for aging effects, which echoed previous findings that RT-related CV loss was progressive $(11,35,36)$. 
Thus, our results suggest that early intervention is necessary to protect temporal cortices during RT, along with previous findings.

In addition to a $\mathrm{CV}$, we also observed time-dependent decreases in CT in the left superior temporal gyrus and the bilateral temporal pole and time-dependent reductions in CSA in the left transverse temporal right inferior temporal gyrus, and the right parahippocampal gyrus. Since $\mathrm{CV}$ is a $\mathrm{CT}$ and CSA product, a reduction in the $\mathrm{CV}$ may reflect either thickness reduction, area atrophy, or both (37). Thus, the CV reduction in the left superior temporal gyrus and the bilateral temporal pole was mainly driven by changes in the CT, while in the left transverse temporal gyrus, the right inferior temporal gyrus, and the right parahippocampal gyrus, reduced $\mathrm{CV}$ was mainly due to CSA atrophy. The time-dependent temporal CT thinning that we have observed in the present study is similar to the observations of CT changes seen in previous studies. For example, in a longitudinal study, temporal lobes have been suggested to be one of the most susceptible to cortical thinning one-year post-RT in brain tumor patients (36). In cross-sectional studies, significant CT reductions involving the temporal pole and inferior temporal gyrus, middle temporal gyrus, and superior temporal gyrus have also been found in post-RT patients within six months following RT administration and in post-RT patients with proven radiation encephalopathy at follow-up (7).

However, the reduction of CSA in the temporal lobe seen in our study was contrary to that seen in a previous cross-sectional study from Zhang et al. (7), who observed a gradual increase in temporal lobe CSA with increasing time after RT within three groups. While such a discrepancy is difficult to explain, to reconcile these contradictory reports, we postulate that the use of absolute CSA as is calculated in a cross-sectional study without a temporal reference lacks definitive value. In contrast, longitudinal changes in CSA within each patient are a more reliable measurement as measured in our study. Our longitudinal study highlights the temporal changes following RT, suggesting that changes in cortical morphology over time may be an indicator of pathological processes in the cerebral cortex after RT.

We also observed time-dependent reduction in cortical morphology (CV/CSA) in the lower part of the occipital (lateral occipital gyrus, lingual gyrus) and frontal lobe (lateral orbitofrontal gyrus). These regions lie relatively close to the basicranium, which may receive unnecessary radiation and result in radioactive damage. The lateral occipital gyrus and lingual gyrus in the occipital region play a critical role in visual information processing $(38,39)$. The lateral occipital gyrus is an important center for social reinforcement learning and emotional regulation (40-42). Impairment of visual processing has been recognized as a prominent clinical feature in NPC patients with radiation-induced injury, and poorer social relationships and higher levels of depression and anxiety have been noted in previous studies in NPC patients after RT $(43,44)$. Thus, we speculate that morphologic abnormalities in these regions may underlie the compromised visual function, social interactions, and emotional changes in NPC patients post-RT.

Importantly, in addition to the time-dependent feature, we also observed dose-dependent alterations in cortical morphology in the temporal (the left fusiform gyrus, parahippocampal gyrus, and superior temporal gyrus, the right transverse temporal gyrus, and inferior temporal gyrus), occipital, and frontal cortices (the right lateral occipital gyrus and lateral occipital gyrus). Taken together, these results show that longitudinal progressive atrophy over time in these brain regions in NPC patients is most likely directly induced by RT, indicating that a safe irradiation dose is necessary to protect these vulnerable cortex regions. The knowledge of RT-related dynamic cortical damage may help illustrate the neurobiology of RTrelated cognitive impairment in NPC patients, which helps establish preventive and protective measurements clinically.

\section{Possible mechanisms for RT-induced reductions in CV, CT, and CSA of the temporal and occipital cortices}

CT and CSA differentially contributed to volume loss. CT is a marker of neuronal integrity in a cortical column, whereas CSA is thought to be a marker of the number and spacing of cortical columns (45). Both these measures have different evolutionary etiologies and genetic determinants $(14,46,47)$. Although the exact biological processes that underlie RT-induced CT and CSA reduction over time in multiple cortices in NPC patients remain unknown, several underlying pathophysiological mechanisms have been proposed. Firstly, CT is influenced by the neuronal size and the number of glial cells, intra-neuronal neuropil, and blood vessels (48). RT-induced injury is now hypothesized to occur due to dynamic interactions between multiple cell types, resulting in atrophy and apoptosis involving oligodendrocytes, subependymal cells, certain types of neurons, endothelial cells, and neural precursor cells $(3,49,50)$. Likely, a reduced number of neurons and glial cells' loss contribute to the diminished CT $(49,51)$. 
Additionally, endothelial damage and apoptosis of oligodendrocytes may result in myelin decrease around neurons that, in turn, can lead to a compromised role of oligodendrocytes in supporting cortical architecture (50), potentially exacerbating cortical thinning. Secondly, the size of the CSA is related to the number of ontogenetic columns. The CSA measures are obtained from the gray-white matter boundary, and it is speculated that CSA atrophy might reflect the cortices and the pathology of underlying white matter fibers (52). This is because the release in tensile forces or disintegration of these fibers could lead to flattened sulci and decreased CSA (16). This speculation is supported by previous diffusion tensor MRI findings in NPC patients after RT $(4,5)$, which revealed FA reductions accompanied by decreased axial diffusivity and increased radial diffusivity in bilateral temporal lobes, indicating axonal damage and demyelination. However, these changes' precise underlying mechanisms must be elucidated in future studies combining MRI-based volumetric measurements and histological evaluation.

\section{Indirect effects of irradiation on the frontal lobe}

To our surprise, we found time-dependent increases in CT in the left rostral middle frontal gyrus of NPC patients during the first year post-RT. The rostral middle frontal gyrus is critical for higher-order executive functions that are associated with stress perception and appraisal $(53,54)$. Recent studies have linked increased CT of the rostral middle frontal gyrus to both severity in depression and stronger perceived stress (53-55). Thus, these timedependent changes to the CT of the left rostral middle frontal gyrus may underlie the higher depression and perceived stress in NPC patients post-RT. This speculation is supported by previous psychological studies (56-58) in NPC patients that demonstrated a time-dependent increase in perceived stress and depression post-RT. If this hypothesis holds, the rostral middle frontal gyrus can be used as a target for the treatment of depression or anxiety in patients with NPC after RT. However, further studies combining longitudinal MRI and detail psychological measurement, especially for depression and perceived stress, are needed to elucidate this question.

\section{Clinical implications}

Our results revealed divergent effects of irradiation on brain cortical morphology that have not been previously reported and the knowledge of which may increase the benefits for NPC patients' clinical management treated with RT. First, the finding that RT-induced deficits in cortical morphology of multiple cortices are progressive during the first year after RT suggests that these cortices may be more sensitive to irradiation than other cortices and are subject to more serious injury in the late-delayed stage. Therefore, selective dose sparing of these structures during treatment plans and/or early intervention should be considered to prevent or minimize cortices injury in NPC patients receiving RT. Also, the findings of indirect effects of irradiation on the rostral middle frontal gyrus imply that psychological conditions (such as perceived stress and depression) in these patients should be paid more attention, and supportive care measures might be considered if indicated.

\section{Limitation}

Despite this study's strengths in its novelty, relatively large sample size, and the use of an automated SBM analysis method, several limitations need to be recognized. First, more research is needed to determine whether cortical changes after RT are reversible and whether there longterm or delayed cortical damage resulting from this treatment. Second, in addition to RT, most of the NPC patients also received chemotherapy in the present study. Thus, the possibility that chemotherapy affects cortical morphology alterations cannot be ruled out. However, dose-dependent abnormalities in different cortical features were observed in multiple brain subregions after RT. This suggests that these abnormalities are likely due to RT. Future studies should enroll an RT only group to elucidate the chemotherapy-related effects. Third, primary tumor staging has also been reported to be an independent predictive factor for RT-induced brain injury in NPC patients (59). In the future, tumor staging-related effects on RT-induced cortical morphology changes of NPC patients should be studied intensively. Finally, studies, including cognitive and neurological assessments, are also needed further to characterize the cortical morphology alterations' clinical significance. These studies will help prioritize anatomical targets for intervention.

\section{Conclusions}

Using an SBM approach, this longitudinal study 
characterized the changes in brain cortical morphology over time in a relatively large sample of patients with NPC during a one-year follow-up after RT. In NPC patients treated with RT, significant and progressive RT-associated decreases of CV, CT, and CSA were detected mainly in the temporal, basal occipital, and basal frontal lobes. These effects on multiple cortices were dose-dependent, which indicates that cortex dose sparing during irradiation is necessary for these vulnerable regions. Also, the timedependent increase in CT in the left rostral middle frontal gyrus that we observed might be associated with depression and perceived stress seen in NPC patients. Our preliminary findings of divergent effects of irradiation on brain cortical morphology may guide us for investigating critically neuroprotective strategies intended to prevent and minimize RT-related damage to the cerebral cortex. Furthermore, these findings may reveal macroscopic changes to guide the investigation of the underlying mechanisms that lead to these neurotoxic effects.

\section{Acknowledgments}

Funding: This work was funded by grants from the Natural Scientific Foundation of China (grant numbers: 81401399, 81560283, and 81201084), the Guangdong Basic and Applied Basic Research Foundation (2019A1515011143).

\section{Footnote}

Conflicts of Interest: All authors have completed the ICMJE uniform disclosure form (available at http://dx.doi. org/10.21037/qims-20-662). The authors have no conflicts of interest to declare.

Ethical Statement: The study was approved by the Institutional Review Board of Sun Yat-sen University Cancer Center (approval number, GZR2014-073) and informed consent was obtained from all individual participants included in the study.

Open Access Statement: This is an Open Access article distributed in accordance with the Creative Commons Attribution-NonCommercial-NoDerivs 4.0 International License (CC BY-NC-ND 4.0), which permits the noncommercial replication and distribution of the article with the strict proviso that no changes or edits are made and the original work is properly cited (including links to both the formal publication through the relevant DOI and the license). See: https://creativecommons.org/licenses/by-nc-nd/4.0/.

\section{References}

1. Bray F, Ferlay J, Soerjomataram I, Siegel RL, Torre LA, Jemal A. Global cancer statistics 2018: GLOBOCAN estimates of incidence and mortality worldwide for 36 cancers in 185 countries. CA Cancer J Clin 2018;68:394-424.

2. Lee AW, Ma BB, Ng WT, Chan AT. Management of Nasopharyngeal Carcinoma: Current Practice and Future Perspective. J Clin Oncol 2015;33:3356-64.

3. Makale MT, Mcdonald CR, Hattangadi-Gluth JA, Kesari S. Mechanisms of radiotherapy-associated cognitive disability in patients with brain tumours. Nat Rev Neurol 2017;13:52-64.

4. Wang HZ, Qiu SJ, Lv XF, Wang YY, Liang Y, Xiong WF, Ouyang ZB. Diffusion tensor imaging and 1H-MRS study on radiation-induced brain injury after nasopharyngeal carcinoma radiotherapy. Clin Radiol 2012;67:340-45.

5. Xiong WF, Qiu SJ, Wang HZ, Lv XF. 1H-MR spectroscopy and diffusion tensor imaging of normalappearing temporal white matter in patients with nasopharyngeal carcinoma after irradiation: initial experience. J Magn Reson Imaging 2013;37:101-08.

6. Leng X, Fang P, Lin H, An J, Tan X, Zhang C, Wu D, Shen W, Qiu S. Structural MRI research in patients with nasopharyngeal carcinoma following radiotherapy: A DTI and VBM study. Oncol Lett 2017;14:6091-96.

7. Zhang YM, Chen MN, Yi XP, Li L, Gao JM, Zhang JL, Yuan XR, Zhang N, Liu LZ, Cai PQ, Chen BT, Zee C, Liao WH, Zhang YC. Cortical Surface Area Rather Than Cortical Thickness Potentially Differentiates Radiation Encephalopathy at Early Stage in Patients With Nasopharyngeal Carcinoma. Front Neurosci 2018;12:599.

8. Lin J, Lv X, Niu M, Liu L, Chen J, Xie F, Zhong M, Qiu S, Li L, Huang R. Radiation-induced abnormal cortical thickness in patients with nasopharyngeal carcinoma after radiotherapy. Neuroimage Clin 2017;14:610-21.

9. Lv XF, Zheng XL, Zhang WD, Liu LZ, Zhang YM, Chen MY, Li L. Radiation-induced changes in normal-appearing gray matter in patients with nasopharyngeal carcinoma: a magnetic resonance imaging voxel-based morphometry study. Neuroradiology 2014;56:423-30.

10. Shi L, Du FL, Sun ZW, Zhang L, Chen YY, Xie TM, Li PJ, Huang S, Dong BQ, Zhang MM. Radiation-induced 
gray matter atrophy in patients with nasopharyngeal carcinoma after intensity modulated radiotherapy: a MRI magnetic resonance imaging voxel-based morphometry study. Quant Imaging Med Surg 2018;8:902-09.

11. Guo Z, Han L, Yang Y, He H, Li J, Chen H, Song T, Qiu Y, Lv X. Longitudinal brain structural alterations in patients with nasopharyngeal carcinoma early after radiotherapy. Neuroimage Clin 2018;19:252-59.

12. Palaniyappan L, Liddle PF. Differential effects of surface area, gyrification and cortical thickness on voxel based morphometric deficits in schizophrenia. Neuroimage 2012;60:693-99.

13. Singh V, Chertkow H, Lerch JP, Evans AC, Dorr AE, Kabani NJ. Spatial patterns of cortical thinning in mild cognitive impairment and Alzheimer's disease. Brain 2006;129:2885-93.

14. Panizzon MS, Fennema-Notestine C, Eyler LT, Jernigan TL, Prom-Wormley E, Neale M, Jacobson K, Lyons MJ, Grant MD, Franz CE, Xian H, Tsuang M, Fischl B, Seidman L, Dale A, Kremen WS. Distinct genetic influences on cortical surface area and cortical thickness. Cereb Cortex 2009; 19:2728-35.

15. Wang D, Han L, Xi C, Xu Y, Lai J, Lu S, Huang M, Hu J, Wei N, Xu W, Zhou W, Lu Q, He H, Hu S. Interactive effects of gender and sexual orientation on cortical thickness, surface area and gray matter volume: a structural brain MRI study. Quant Imaging Med Surg 2020;10:835-46.

16. Carmona S, Martinez-Garcia M, Paternina-Die M, BarbaMuller E, Wierenga LM, Aleman-Gomez Y, Pretus C, Marcos-Vidal L, Beumala L, Cortizo R, Pozzobon C, Picado M, Lucco F, Garcia-Garcia D, Soliva JC, Tobena A, Peper JS, Crone EA, Ballesteros A, Vilarroya O, Desco M, Hoekzema E. Pregnancy and adolescence entail similar neuroanatomical adaptations: A comparative analysis of cerebral morphometric changes. Hum Brain Mapp 2019;40:2143-52.

17. Seibert TM, Karunamuni R, Kaifi S, Burkeen J, Connor M, Krishnan AP, White NS, Farid N, Bartsch H, Murzin V, Nguyen TT, Moiseenko V, Brewer JB, Mcdonald CR, Dale AM, Hattangadi-Gluth JA. Cerebral Cortex Regions Selectively Vulnerable to Radiation Dose-Dependent Atrophy. Int J Radiat Oncol Biol Phys 2017;97:910-18.

18. Edge SB, Byrd DR, Compton CC, Fritz AG, Greene FL, Trotti A. AJCC cancer staging manual (7th ed). New York: Springer, 2010.

19. Sun Y, Yu XL, Luo W, Lee AW, Wee JT, Lee N, Zhou GQ, Tang LL, Tao CJ, Guo R, Mao YP, Zhang R, Guo Y,
Ma J. Recommendation for a contouring method and atlas of organs at risk in nasopharyngeal carcinoma patients receiving intensity-modulated radiotherapy. Radiother Oncol 2014;110:390-97.

20. Zeng L, Huang SM, Tian YM, Sun XM, Han F, Lu TX, Deng XW. Normal Tissue Complication Probability Model for Radiation-induced Temporal Lobe Injury after Intensity-modulated Radiation Therapy for Nasopharyngeal Carcinoma. Radiology 2015;276:243-49.

21. Yang Y, Lin X, Li J, Han L, Li Z, Liu S, Hou G, Xie C, Lv X, Qiu Y. Aberrant Brain Activity at Early Delay Stage Post-radiotherapy as a Biomarker for Predicting Neurocognitive Dysfunction Late-Delayed in Patients With Nasopharyngeal Carcinoma. Front Neurol 2019;10:752.

22. Dale AM, Fischl B, Sereno MI. Cortical surface-based analysis. I. Segmentation and surface reconstruction. Neuroimage 1999;9:179-94.

23. Fischl B, Salat DH, Busa E, Albert M, Dieterich M, Haselgrove C, van der Kouwe A, Killiany R, Kennedy D, Klaveness S, Montillo A, Makris N, Rosen B, Dale AM. Whole brain segmentation: automated labeling of neuroanatomical structures in the human brain. Neuron 2002;33:341-55.

24. Fischl B, Sereno MI, Dale AM. Cortical surface-based analysis. II: Inflation, flattening, and a surface-based coordinate system. Neuroimage 1999;9:195-207.

25. Fischl B, van der Kouwe A, Destrieux C, Halgren E, Segonne F, Salat DH, Busa E, Seidman LJ, Goldstein J, Kennedy D, Caviness V, Makris N, Rosen B, Dale AM. Automatically parcellating the human cerebral cortex. Cereb Cortex 2004;14:11-22.

26. Lv X, He H, Yang Y, Han L, Guo Z, Chen H, Li J, Qiu Y, Xie C. Radiation-induced hippocampal atrophy in patients with nasopharyngeal carcinoma early after radiotherapy: a longitudinal MR-based hippocampal subfield analysis. Brain Imaging Behav 2019;13:1160-71.

27. Reuter M, Schmansky NJ, Rosas HD, Fischl B. Withinsubject template estimation for unbiased longitudinal image analysis. Neuroimage 2012;61:1402-18.

28. Desikan RS, Segonne F, Fischl B, Quinn BT, Dickerson BC, Blacker D, Buckner RL, Dale AM, Maguire RP, Hyman BT, Albert MS, Killiany RJ. An automated labeling system for subdividing the human cerebral cortex on MRI scans into gyral based regions of interest. Neuroimage 2006;31:968-80.

29. Buckner RL, Head D, Parker J, Fotenos AF, Marcus D, Morris JC, Snyder AZ. A unified approach for 
morphometric and functional data analysis in young, old, and demented adults using automated atlas-based head size normalization: reliability and validation against manual measurement of total intracranial volume. Neuroimage 2004;23:724-38.

30. Jack CR, Twomey CK, Zinsmeister AR, Sharbrough FW, Petersen RC, Cascino GD. Anterior temporal lobes and hippocampal formations: normative volumetric measurements from MR images in young adults. Radiology 1989;172:549-54.

31. Ng KK, Qiu Y, Lo JC, Koay ES, Koh WP, Chee MW, Zhou J. Functional segregation loss over time is moderated by APOE genotype in healthy elderly. Hum Brain Mapp 2018;39:2742-52.

32. Singer J, Willett J. Applied longitudinal data analysis: Modeling change and event occurrence. New York: Oxford University Press, 2003.

33. Cnaan A, Laird NM, Slasor P. Using the general linear mixed model to analyse unbalanced repeated measures and longitudinal data. Stat Med 1997;16:2349-80.

34. Long J. Longitudinal Data Analysis for the Behavioral Sciences Using R. CA: Sage, Thousand Oaks, 2012.

35. Gazdzinski LM, Cormier K, Lu FG, Lerch JP, Wong CS, Nieman BJ. Radiation-induced alterations in mouse brain development characterized by magnetic resonance imaging. Int J Radiat Oncol Biol Phys 2012;84:e631-38.

36. Karunamuni R, Bartsch H, White NS, Moiseenko V, Carmona R, Marshall DC, Seibert TM, Mcdonald CR, Farid N, Krishnan A, Kuperman J, Mell L, Brewer JB, Dale AM, Hattangadi-Gluth JA. Dose-Dependent Cortical Thinning After Partial Brain Irradiation in High-Grade Glioma. Int J Radiat Oncol Biol Phys 2016;94:297-304.

37. Vijayakumar N, Allen NB, Youssef G, Dennison M, Yucel M, Simmons JG, Whittle S. Brain development during adolescence: A mixed-longitudinal investigation of cortical thickness, surface area, and volume. Hum Brain Mapp 2016;37:2027-38.

38. Nagy K, Greenlee MW, Kovacs G. The lateral occipital cortex in the face perception network: an effective connectivity study. Front Psychol 2012;3:141.

39. Lee HW, Hong SB, Seo DW, Tae WS, Hong SC. Mapping of functional organization in human visual cortex: electrical cortical stimulation. Neurology 2000;54:849-54.

40. Ross RS, Lopresti ML, Schon K, Stern CE. Role of the hippocampus and orbitofrontal cortex during the disambiguation of social cues in working memory. Cogn Affect Behav Neurosci 2013;13:900-15.

41. Petrovic P, Ekman CJ, Klahr J, Tigerstrom L, Ryden G,
Johansson AG, Sellgren C, Golkar A, Olsson A, Ohman A, Ingvar M, Landen M. Significant grey matter changes in a region of the orbitofrontal cortex in healthy participants predicts emotional dysregulation. Soc Cogn Affect Neurosci 2016;11:1041-49.

42. Rolls ET. The functions of the orbitofrontal cortex. Brain Cogn 2004;5 5:11-29.

43. Mo YL, Li L, Qin L, Zhu XD, Qu S, Liang X, Wei ZJ. Cognitive function, mood, and sleep quality in patients treated with intensity-modulated radiation therapy for nasopharyngeal cancer: a prospective study. Psychooncology 2014;23:1185-91.

44. Tang Y, Luo D, Rong X, Shi X, Peng Y. Psychological disorders, cognitive dysfunction and quality of life in nasopharyngeal carcinoma patients with radiation-induced brain injury. Plos One 2012;7:e36529.

45. Rakic P. Specification of cerebral cortical areas. Science 1988;241:170-76.

46. Tamnes CK, Herting MM, Goddings AL, Meuwese R, Blakemore SJ, Dahl RE, Guroglu B, Raznahan A, Sowell ER, Crone EA, Mills KL. Development of the Cerebral Cortex across Adolescence: A Multisample Study of InterRelated Longitudinal Changes in Cortical Volume, Surface Area, and Thickness. J Neurosci 2017;37:3402-12 .

47. Geschwind DH, Rakic P. Cortical evolution: judge the brain by its cover. Neuron 2013;80:633-47.

48. Rakic P. A small step for the cell, a giant leap for mankind: a hypothesis of neocortical expansion during evolution. Trends Neurosci 1995;18:383-88.

49. Yang L, Yang J, Li G, Li Y, Wu R, Cheng J, Tang Y. Pathophysiological Responses in Rat and Mouse Models of Radiation-Induced Brain Injury. Mol Neurobiol 2017;54:1022-32.

50. Greene-Schloesser D, Robbins ME, Peiffer AM, Shaw EG, Wheeler KT, Chan MD. Radiation-induced brain injury: A review. Front Oncol 2012;2:73.

51. Nagtegaal SH, David S, van der Boog A, Leemans A, Verhoeff J. Changes in cortical thickness and volume after cranial radiation treatment: A systematic review. Radiother Oncol 2019;135:33-42.

52. Van Essen DC. A tension-based theory of morphogenesis and compact wiring in the central nervous system. Nature 1997;385:313-18.

53. Koenigs M, Grafman J. The functional neuroanatomy of depression: distinct roles for ventromedial and dorsolateral prefrontal cortex. Behav Brain Res 2009;201:239-43.

54. Michalski LJ, Demers CH, Baranger D, Barch DM, Harms MP, Burgess GC, Bogdan R. Perceived stress is 
associated with increased rostral middle frontal gyrus cortical thickness: a family-based and discordant-sibling investigation. Genes Brain Behav 2017;16:781-89.

55. Phillips JL, Batten LA, Tremblay P, Aldosary F, Blier P. A Prospective, Longitudinal Study of the Effect of Remission on Cortical Thickness and Hippocampal Volume in Patients with Treatment-Resistant Depression. Int J Neuropsychopharmacol 2015;18:pyv037.

56. Reynolds S, Carrey N, Jaworska N, Langevin LM, Yang XR, Macmaster FP. Cortical thickness in youth with major depressive disorder. BMC Psychiatry 2014;14:83.

57. Lee PW, Kwan TT, Kwong DL, Sham JS, Pow EH, Mcmillan AS, Au GK. A prospective study of the

Cite this article as: $\mathrm{Lv} \mathrm{X}$, Guo Z, Tang L, Li Z, Lin X, Li J, Han L, Qiu Y, Mai H. Divergent effects of irradiation on brain cortical morphology in patients with nasopharyngeal carcinoma: one-year follow-up study using structural magnetic resonance imaging. Quant Imaging Med Surg 2021;11(6):2307-2320. doi: 10.21037/qims-20-662 impact of nasopharyngeal cancer and radiotherapy on the psychosocial condition of Chinese patients. Cancer 2007;109:1344-54.

58. Chen AM, Jennelle RL, Grady V, Tovar A, Bowen K, Simonin P, Tracy J, Mccrudden D, Stella JR, Vijayakumar S. Prospective study of psychosocial distress among patients undergoing radiotherapy for head and neck cancer. Int J Radiat Oncol Biol Phys 2009;73:187-93.

59. Feng M, Huang Y, Fan X, Xu P, Lang J, Wang D. Prognostic variables for temporal lobe injury after intensity modulated-radiotherapy of nasopharyngeal carcinoma. Cancer Med 2018;7:557-64. 


\section{Appendix 1: Treatment}

Target volumes were delineated slice - by - slice on CT images for treatment planning using an individualized delineation protocol. The prescribed dose was 68-70 Gy, 60-70 Gy, 60-64 Gy, and 54-58 Gy for the planning target volumes (PTVs) derived from the gross tumor volume (GTVnx), GTV of involved lymph nodes (GTVnd), high risk clinical target volume (CTV1), and low - risk clinical target volume (CTV2), respectively. Each patient was treated once daily for 5 days per week, with a total of 30-33 fractions. To calculate the dose-volume statistics for the bilateral frontal, temporal, and occipital lobe, the anatomic boundaries of these regions were manually delineated on axial CT images with T1-weighted images as anatomical reference after treatment planning.
In addition, during the study, according to the institutional guidelines, treatment regimens that RT only for stage I of NPC and concurrent chemoradiotherapy with or without neoadjuvant/adjuvant chemotherapy for stages II to IVB of NPC were recommended. Of the 104 patients, two (1.92\%) underwent only RT, 53 (50.96\%) were administered concurrent chemoradiotherapy, and 49 patients $(47.12 \%)$ received a combination of neoadjuvant and concurrent chemoradiotherapy. Neoadjuvant therapy consisted of cisplatin with 5-fluorouracil (PF), cisplatin with docetaxel (TP), or docetaxel, cisplatin, and 5-fluorouracil and (TPF) every three weeks for $\geq 2$ cycles. The concurrent chemotherapy consisted of cisplatin/nedaplatin or paclitaxel administered weekly for at least 4-7 cycles or in weeks 1, 4, and 7 of radiation therapy.

Table S1 Cortical morphology with significant group $\times$ time interaction in the first model showed time (post-RT) dependent alterations in NPC patients (IMRT only) during the first year post RT, corrected for total intracranial volume (TIV), age, education, and gender in patients with nasopharyngeal carcinoma

\begin{tabular}{|c|c|c|}
\hline Brain region & Coefficient & $P$ \\
\hline CV_STG.L & -70.04 & $2.50 \mathrm{E}-11^{*}$ \\
\hline CV_STG.R & -66.9 & $2.60 \mathrm{E}-11^{*}$ \\
\hline CV_MTG.L & -72.7 & $5.68 \mathrm{E}-10^{*}$ \\
\hline CV_MTG.R & -78.3 & $1.85 \mathrm{E}-9^{\star}$ \\
\hline CV_TP.L & -26.8 & $7.07 \mathrm{E}-13^{*}$ \\
\hline CV_TP.R & -34.3 & $2.22 \mathrm{E}-16^{*}$ \\
\hline CV_PHG.L & -14.1 & $2.48 \mathrm{E}-08^{*}$ \\
\hline CV_PHG.R & -14.3 & $9.65 \mathrm{E}-08^{*}$ \\
\hline CV_ITG.R & -95.5 & $7.09 \mathrm{E}-13^{*}$ \\
\hline CV_TTG.R & -9.01 & $1.61 \mathrm{E}-11^{*}$ \\
\hline CV_FG.L & -56.8 & $1.57 \mathrm{E}-08^{*}$ \\
\hline CV_FG.R & -62.1 & $4.91 \mathrm{E}-10^{*}$ \\
\hline CV_LOG.R & -102.4 & $2.81 \mathrm{E}-07^{*}$ \\
\hline CV_LOFG.R & -58 & $1.15 \mathrm{E}-06^{*}$ \\
\hline CT_TP.L & -0.02 & $6.20 \mathrm{E}-05^{*}$ \\
\hline CT_TP.R & -0.02 & $2.88 \mathrm{E}-05^{*}$ \\
\hline CT_STG.L & -0.01 & $7.87 \mathrm{E}-12^{*}$ \\
\hline CT_RMFG.L & 0.01 & $5.65 \mathrm{E}-07^{*}$ \\
\hline CSA_TTG.L & -3.72 & $4.57 \mathrm{E}-12^{*}$ \\
\hline CSA_ITG.R & -11.3 & $2.00 \mathrm{E}-10^{*}$ \\
\hline CSA_LG.R & -19.4 & $5.81 \mathrm{E}-11^{*}$ \\
\hline CSA_PHG.R & -2.35 & $3.21 \mathrm{E}-07^{*}$ \\
\hline
\end{tabular}

*Statistically significant effects $(P<0.05)$. CV, cortical volume; $C T$, cortical thickness; CSA, cortical surface area; STG, superior temporal gyrus; MTG, middle temporal gyrus; TP, temporal pole; PHG, parahippocampal gyrus; ITG, inferior temporal gyrus; FG, fusiform gyrus; LOG, lateral occipital gyrus; LOFG, lateral orbitofrontal gyrus; RMFG, rostral middle frontal gyrus; TTG, transverse temporal gyrus; LG, lingual gyrus; L, left; R, right. 